\title{
Elementary Particles of Conventional Field Theory as Regge Poles. IV*
}

\author{
M. Gell-Mann, $\dagger$ M. L. Goldberger, $\ddagger$ and F. E. Low \\ Department of Physics and Laboratory of Nuclear Science, Massachusetts Institute of Technology, \\ Cambridge, Massachusetts \\ AND \\ V. Singh§ AND F. Zachariasen \\ California Institute of Technology, Pasadena, California
}

(Received 15 August 1963)

\begin{abstract}
The usual field theory of spin 0 "nucleons" coupled to vector mesons (or heavy photons) is studied in order to find out whether the nucleon lies on a Regge trajectory. Photon-nucleon scattering is examined, to each order in the coupling constant, with the highest power of $\ln \cos \theta$ retained. It is found that a suitable Regge trajectory is generated, but that the nucleon does not lie on it. The nucleon pole term in the scattering amplitude corresponds to a fixed singularity in angular momentum. The spin 0 "nucleon" thus behaves differently from a particle of spin $\frac{1}{2}$.
\end{abstract}

\section{INTRODUCTION}

$I^{\mathrm{N}}$ $\mathrm{N}$ the previous article ${ }^{1}$ we have seen that an "elementary spinor nucleon turns from a fixed singularity in the $J$ plane into a moving pole as a result of vector meson (or heavy photon) radiative corrections. The problem was studied in the approximation of keeping the highest power of $\ln \cos \theta$ for each power of the coupling strength $\gamma^{2}$. The factoring of the Born approximation was crucial in achieving the result.

Here we study the case of a scalar nucleon in the same approximation. We find that in the usual field theory the fixed singularity remains, because the Born approximation does not factor.

Since there is a nonsense channel with $J=0$ and the quantum numbers of the scalar nucleon, a Regge pole of the right kind is generated with $\alpha \rightarrow 0$ as $\gamma^{2} \rightarrow 0$, but the trajectory does not pass through the nucleon.

An alternative, more complicated field theory can be constructed in which the factoring obtains and the scalar nucleon lies on the trajectory, but such a theory does not seem to be renormalizable in the usual sense.

In Sec. 2 we discuss the scalar nucleon problem by much the same methods applied in II and III to the spinor nucleon. The Born approximation is examined and used as the starting point of an iteration method based on unitarity and analyticity for partial wave amplitudes.

In the subsequent sections, the problem is re-examined from a different point of view, that of asymptotic unitarity for scattering amplitudes. The method of asymptotic unitarity could also be employed for the

* This work supported in part by the U. S. Atomic Energy Commission.

$\dagger$ Permanent address: California Institute of Technology, Pasadena, California.

$\ddagger$ Permanent address: Palmer Physical Laboratory, Princeton University, Princeton, New Jersey.

$\$$ On deputation from Tata Institute of Fundamental Research, Bombay, India. Present address: Institute of Advanced Study, Princeton, New Jersey.

${ }_{1}$ M. Gell-Mann, M. L. Goldberger, F. E. Low, E. Marx, and F. Zachariasen, preceding paper, Phys. Rev. 133, B145 (1964). We refer to that paper as III. spinor problem, and it shows directly how the Feynman diagrams generate Regge poles. Extensive use is made of the Mandelstam representation.

The fourth-order Mandelstam weight functions are first introduced in Sec. 3, but the photon-nucleon scattering problem is not taken up immediately. Instead, a scalar meson is introduced, coupled to the nucleon with coupling parameter $g$. The amplitudes for scalar meson-scalar nucleon scattering (order $g^{2} \gamma^{2}$ ) and for photoproduction of scalar nucleons (order $g \gamma^{3}$ ) are discussed. The full spin complications of photon-scalar nucleon scattering are postponed to Sec. 5 .

The sixth-order amplitude $\left(g^{2} \gamma^{4}\right)$ for scalar mesonscalar nucleon scattering is treated in Sec. 4, in order to illustrate what Feynman diagrams are important in the higher orders and what cancellations occur.

In Sec. 5, the method of asymptotic unitarity is developed and applied to photon-nucleon scattering.

Finally, in Sec. 6, the Feynman diagrams for photonnucleon scattering are examined explicitly with the aid of asymptotic unitarity and it is shown how to extract the highest power of $\ln \cos \theta$ for each power of $\gamma^{2}$. In sixth and higher orders, the vanishing of certain subtraction constants still has to be proved.

\section{SECOND-ORDER CALCULATION AND ANGULAR MOMENTUM BEHAVIOR OF COMPTON SCATTERING}

The discussion in this section is parallel to that in Secs. 4 and 5 of III. The second-order Feynman amplitude and scattering amplitude $f$ are given by

$$
M=\epsilon_{2 \mu} \epsilon_{1 \nu} \mathfrak{T T}_{\mu \nu}
$$

and $8 \pi W f=M$, where

$$
\begin{aligned}
\mathfrak{T T}_{\mu \nu}=\gamma^{2}\left\{\frac{\left(2 p_{2 \mu}+k_{2 \mu}\right)\left(2 p_{1 \nu}+k_{1 \nu}\right)}{m^{2}-s}\right. \\
\left.+\frac{\left(2 p_{1 \mu}-k_{2 \mu}\right)\left(2 p_{2 \nu}-k_{1 \nu}\right)}{m^{2}-u}-2 \delta_{\mu \nu}\right\} .
\end{aligned}
$$


As in III, we work in the gauge

$$
\begin{aligned}
& \epsilon_{2}=\epsilon_{2 L}-\left[\left(\epsilon_{2 L} \cdot k_{1} k_{2}\right) /\left(k_{1} \cdot k_{2}\right)\right], \\
& \epsilon_{1}=\epsilon_{1 L}-\left[\left(\epsilon_{1 L} \cdot k_{2} k_{1}\right) /\left(k_{1} \cdot k_{2}\right)\right],
\end{aligned}
$$

where $\epsilon_{L}$ is the Lorentz gauge $\left(\epsilon_{L} \cdot k=0\right)$. Then in the high $t$ limit, the middle term of (2.2) does not contribute to the $f$ amplitudes. The others are easily calculated, using the definitions given in III, Sec. 2:

$$
\begin{aligned}
& 8 \pi W f_{00}+=-\frac{2 \gamma^{2} \lambda^{2}}{\omega^{2}} \frac{\left[\left(s-m^{2} / k\right)-k\right]^{2}}{s-m^{2}}+\frac{4 \gamma^{2} \lambda^{2}}{k^{2}} \\
& 8 \pi W f_{10}{ }^{+}=-\gamma^{2}-\left(\frac{s-m^{2}}{\omega}-k\right) \frac{\sqrt{2}}{k z}+\frac{2 \sqrt{2} \gamma^{2} \omega}{k^{2} z} \\
& 8 \pi W f_{11}{ }^{+}=\frac{\gamma^{2}\left(m^{2}-s\right)}{k^{2} z}+\frac{2 \gamma^{2} \omega^{2}}{k^{2}} \cdot \frac{1}{z} \\
& 8 \pi W f_{11}-=0-\frac{2 \gamma^{2}}{z},
\end{aligned}
$$

where the first and second terms of (2.4) come, in our gauge, from the first and third terms of (2.2), respectively. The motivation for this separation will emerge presently.

The partial wave amplitudes near $J=0$ are then evidently

$$
\begin{aligned}
& 8 \pi W F_{00} J+=-2 \gamma^{2} \frac{\lambda^{2}}{\omega^{2}} \frac{\left\{\left[\left(s-m^{2}\right) / k\right]-k\right\}^{2}}{s-m^{2}} \delta_{J 0}+4 \gamma^{2}{\frac{\lambda}{k^{2}}}_{\delta_{J 0}}, \\
& \frac{8 \pi W F_{10}{ }^{J+}}{J^{1 / 2}}=\gamma^{2}-\left(\frac{s-m^{2}}{k}-k\right) \frac{\sqrt{2}}{k} \cdot \frac{1}{J}-2 \sqrt{2} \frac{\gamma^{2} \lambda \omega}{k^{2}} \frac{1}{J}, \\
& 8 \pi W F_{11}^{J+}=\gamma^{2} \frac{\left(s-m^{2}\right)}{k^{2}} \cdot \frac{1}{J}-\frac{2 \gamma^{2} \omega^{2}}{k^{2}} \cdot \frac{1}{J}, \\
& 8 \pi W F_{11} J-=0+2 \gamma^{2} \cdot \frac{1}{J} .
\end{aligned}
$$

We observe that the two sets of terms factor separately. That is,

$$
\begin{aligned}
& F_{00}{ }^{J+}=-\xi_{0}{ }^{2} \delta_{J 0}+\xi_{0}{ }^{\prime 2} \delta_{J 0}, \\
& \frac{F_{10}{ }^{J+}}{J^{1 / 2}}=-\xi_{0} \xi_{1} \frac{1}{J}+\xi_{0}{ }^{\prime} \xi_{1}^{\prime} \frac{1}{J}, \\
& F_{11}{ }^{J+}=\xi_{1}{ }^{2} / J-\xi_{1}^{\prime 2} / J, \\
& F_{11}^{J-}=0+\gamma^{2} / 4 \pi W J,
\end{aligned}
$$

where

$$
\begin{gathered}
\xi_{1}=\left[\gamma^{2}\left(s-m^{2}\right) / 8 \pi k^{2} W\right]^{1 / 2}, \\
\xi_{0}=\left[\frac{2 \gamma^{2} \lambda^{2}\left\{\left[\left(s-m^{2}\right) / k\right]-k\right\}^{2}}{8 \pi W \omega^{2}\left(s-m^{2}\right)}\right]^{1 / 2}, \\
\xi_{1}^{\prime}=\left[2 \gamma^{2} \omega^{2} / 8 \pi W k^{2}\right]^{1 / 2}, \\
\xi_{0}^{\prime}=\left[4 \gamma^{2} \lambda^{2} / 8 \pi W k^{2}\right]^{1 / 2} .
\end{gathered}
$$

We now observe that the first and second columns factor separately, but that their sum does not, since $\xi_{0} / \xi_{1} \neq \xi_{0}^{\prime} / \xi_{1}^{\prime}$.

Therefore, in this theory, the nucleon does not lie on a trajectory; it fails to become Reggeistic. Instead, we have the following behavior: The negative parity amplitude $F_{11}{ }^{J-}$ is not coupled by unitarity to the other three states, and thus generates, according to the work of III, Sec. 3, a trajectory whose imaginary part is given by

$$
\operatorname{Im} \alpha^{-}=\gamma^{2} k / 4 \pi W \text {. }
$$

The positive parity states generate a trajectory with

$$
\operatorname{Im} \alpha^{+}=k\left(\xi_{1}^{2}-\xi_{1}^{\prime 2}\right)=\gamma^{2}\left(s-m^{2}-2 \omega^{2}\right) / 8 \pi k W,
$$

which, as observed above, does not pass through the nucleon. Further, near $J=\alpha$,

$$
\begin{aligned}
& F_{11}^{J+}=(1 / k)\left[\operatorname{Im} \alpha^{+} /(J-\alpha)\right], \\
& \frac{F_{10}{ }^{J+}}{J}=-\frac{\left(\xi_{0} \xi_{1}-\xi_{0}{ }^{\prime} \xi_{1}{ }^{\prime}\right)}{J-\alpha^{+}}=-\frac{\left(\xi_{0} \xi_{1}-\xi_{0}{ }^{\prime} \xi_{1}{ }^{\prime}\right)}{\left(\operatorname{Im} \alpha^{+} / k\right)^{1 / 2}} \frac{\left(\operatorname{Im} \alpha^{+} / k\right)^{1 / 2}}{J-\alpha^{+}},
\end{aligned}
$$

and

$$
\operatorname{Im} F_{00}{ }^{J+}=\operatorname{Im}\left[\frac{\left(\xi_{0} \xi_{1}-\xi_{0}{ }^{\prime} \xi_{1}{ }^{\prime}\right)^{2}}{\operatorname{Im} \alpha^{+} / k} \frac{\alpha^{+}}{J-\alpha^{+}}\right]
$$

In this case, we cannot simply remove the Im from the last equation above, since the Born approximation limit, where $\alpha^{+} /\left(J-\alpha^{+}\right) \rightarrow \delta_{J 0}$, would then be in disagreement with Eq. (2.6). Evidently, we achieve agreement by writing

$F_{00} J+=\frac{\left(\xi_{0} \xi_{1}-\xi_{0}{ }^{\prime} \xi_{1}\right)^{2}}{\operatorname{Im} \alpha^{+} / k} \frac{\alpha^{+}}{J-\alpha^{+}}+\frac{\left(\xi_{0}{ }^{\prime} \xi_{1}-\xi_{0} \xi_{1}{ }^{\prime}\right)^{2}}{\operatorname{Im} \alpha^{+} / k} \delta_{J_{0}}$,

which, as $\alpha \rightarrow 0$, approaches

$$
\frac{-\left(\xi_{0} \xi_{1}-\xi_{0}{ }^{\prime} \xi_{1}{ }^{\prime}\right)^{2}+\left(\xi_{0}{ }^{\prime} \xi_{1}-\xi_{0} \xi_{1}{ }^{\prime}\right)^{2}}{\operatorname{Im} \alpha^{+} / k} \cdot \delta_{J 0}=\delta_{J 0}\left(-\xi_{0}{ }^{2}+\xi_{0}{ }^{2}\right)
$$

in agreement with (2.6).

We therefore find trajectories in the neighborhood of $J=0$, which, however, do not pass through the nucleon exactly at $J=0$. Consequently, the nucleon remains unReggeized, and the nucleon pole is completely contained in the coefficient of $\delta_{J 0}$ in (2.11). 
The asymptotic behavior of the f's in $z$ may now be predicted. It is evidently

$$
\begin{aligned}
& f_{00}{ }^{+}=\frac{\left(\xi_{0}{ }^{\prime} \xi_{1}-\xi_{0} \xi_{1}{ }^{\prime}\right)^{2}}{\operatorname{Im} \alpha^{+} / k}-\frac{\left(\xi_{0} \xi_{1}-\xi_{0}{ }^{\prime} \xi_{1}\right)^{2}}{\operatorname{Im} \alpha^{+} / k}(-z)^{\alpha^{+}} \\
& f_{10}{ }^{+}=\left(\xi_{0} \xi_{1}-\xi_{0}{ }^{\prime} \xi_{1}{ }^{\prime}\right)\left[(-z)^{\alpha^{+}} / z\right], \\
& f_{11^{+}}=-\left(\xi_{0}{ }^{2}-\xi_{1}{ }^{2}\right)\left[(-z)^{\alpha^{+}} / z\right] \\
& f_{11}{ }^{-}=-\left(\gamma^{2} / 4 \pi W\right)\left[(-z)^{\alpha^{-}} / z\right] .
\end{aligned}
$$

It is interesting to observe that the theory without the $\delta_{\mu \nu}$ term (in our gauge) does Reggeize the nucleon; one has in that case $F_{11}{ }^{J-}=0$, and

$$
\begin{aligned}
& F_{11}{ }^{J+}=\xi_{1}{ }^{2} /(J-\alpha), \\
& F_{10}{ }^{J+}=-\xi_{0} \xi_{1}(J)^{1 / 2} /(J-\alpha), \\
& F_{00}{ }^{J+}=\xi_{0}^{2}[\alpha /(J-\alpha)],
\end{aligned}
$$

with $\operatorname{Im} \alpha=k \xi_{1}^{2}=\gamma^{2}\left(s-m^{2}\right) / 8 \pi k^{2} W$, so that $\operatorname{Im} \alpha=0$ at $s=m^{2}$.

We may then ask if there exists a theory which would yield precisely these results, that is, would not have the " $\delta_{\mu \nu}$ term," which in our gauge becomes

$$
-2 \gamma^{2}\left[\delta_{\mu \nu} k_{1} \cdot k_{2}-k_{1 \mu} k_{2 \nu}\right] /\left(k_{1} \cdot k_{2}\right) \text {. }
$$

Since our whole treatment has been concerned with large $z$, i.e., large $k_{1} \cdot k_{2}$, we may modify this expression by terms which are uninteresting in such a limit; for example, we write

$$
+4 \gamma^{2} \frac{\delta_{\mu \nu} k_{1} \cdot k_{2}-k_{1 \mu} k_{2 \nu}}{\left(k_{1}-k_{2}\right)^{2}+M^{2}},
$$

where $M$ is an arbitrary mass. If there were in the Lagrangian an interaction of the form

$$
\mathfrak{L}_{I}=\gamma_{1} F_{\alpha \beta} F_{\alpha \beta} \chi+\gamma_{2} \phi^{\dagger} \phi \chi,
$$

where $F_{\alpha \beta}$ is the field strength associated with our vector field, $\chi$ is a new scalar field of mass $M$, and $\gamma_{1} \gamma_{2}=-\gamma^{2}$, then the contribution (2.15) to the Compton scattering would be cancelled by the Born approximation exchange of a single $\chi$ particle.

The modified theory consisting of the normal interaction between the scalar nucleon and vector meson fields as well as the new interaction, Eq. (2.16), is not obviously renormalizable. In the case of real photons we note that the electromagnetic interaction would no longer be minimal.

\section{FOURTH-ORDER SCATTTERING AND PHOTOPRODUCTION}

In this section we begin a discussion of the entire problem from the point of view of Mandelstam weight functions and asymptotic unitarity. The results will be equivalent to those of Sec. 2. The new treatment is intended to clarify certain points, such as the absence
Fig. 1. Feynman graphs for fourth-order mesonnucleon scattering, contributing to the double spectral functions. Mesons are wiggly, nucleons are $P$ solid, and vector mesons are dashed lines.
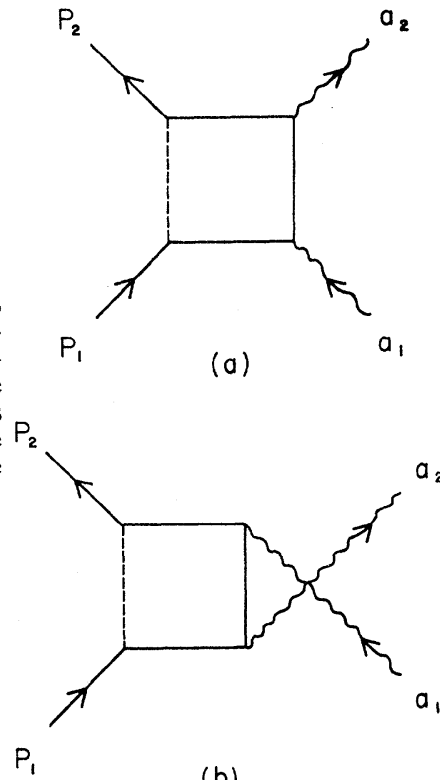

(b)

of left-hand $s$ cuts in the leading terms. In addition, it may be helpful to the reader to have an alternative approach. We start with an explicit discussion of the lowest few orders of perturbation theory. Fourth order is described here; sixth order is deferred to the following section.

We take up first the study of meson-nucleon scattering, with spinless mesons and nucleons, to minimize the complexity. It will, of course, not be possible to eliminate all vestiges of spinology, and still have the possibility of Regge behavior of the nucleon, so we assume the existence of virtual vector mesons coupled to the nucleon. These will be neutral and interact with a conserved current so that we are dealing with a renormalizable theory in the usual sense. At the end of this section, we shall remark on the fourth-order photoproduction process as well (by which we mean meson $+N \rightarrow N+$ vector meson; to avoid infrared problems, we do not wish, generally, to deal with actual photons). Some information about this is necessary to evaluate the sixth-order scattering process.

Meson-nucleon scattering is described by a single invariant scattering amplitude, which we may call $\Re(s, t)$; it depends on $s=-\left(p_{1}+q_{1}\right)^{2}$ and $t=-\left(p_{1}-p_{2}\right)^{2}$, where $p_{1}\left(p_{2}\right)$ and $q_{1}\left(q_{2}\right)$ are the four-momenta of the initial (final) nucleon and meson, respectively. We shall study the behavior of $\mathfrak{N}(s, t)$ at large $t$ for fixed $s$.

In fourth order there are eight Feynman diagrams contributing to $\mathfrak{M}$; two of these are shown in Fig. 1. Evidently, Fig. 1(a) is obtained from Fig. 1(b) by replacing $s$ by $u$, where $u=4-s-t$. (We shall from now on take the nucleon, meson, and vector-meson masses equal, and equal to unity, to keep the formulas as simple as possible. It is not a great amount of extra labor to keep them all different, as is shown in Sec. 2, but, in 

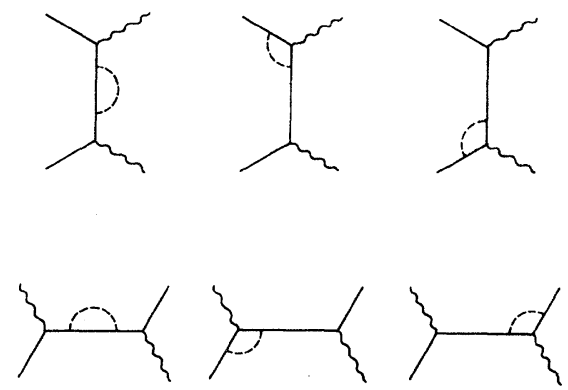

FIG. 2. Fourth-order meson-nucleon scattering "renormalization" graphs.

general, nothing is particularly clarified by doing so.) The remaining graphs, in fourth order, are all "renormalization graphs" and depend only on $s$ or on $u$. They are shown in Fig. 2.

Using the usual Cutkosky rules, ${ }^{2}$ it is quite straightforward to compute the double spectral function for the graph of Fig. 1(a); the result is

$$
\begin{aligned}
\rho^{13}(s, t) \rightarrow-\frac{1}{8} g^{2} \gamma^{2} \frac{(3-2 t)}{[s(s-4) t(t-4)-4 s t]^{1 / 2}} \\
\equiv(3-2 t) \rho_{0}(s, t) .
\end{aligned}
$$

Here the meson-nucleon vertex is taken as $g$ and the vector meson nucleon vertex is $\gamma\left(p_{1}+p_{2}\right)_{\mu}$, when a vector meson of polarization $\mu$ is absorbed by a nucleon of momentum $p_{1}$, giving a nucleon of momentum $p_{2}$. The crossed box, Fig. 1(b), contributes the same, with $s \rightarrow u$, to the spectral function $\rho^{23}(u, t)$. Clearly, computing $\mathfrak{T}$ from $\rho^{13}$ gives a term like $\ln t$ for large $t$; the contribution of $\rho^{23}$ is like $(\ln t)^{2} / t$. The renormalization graphs in $s$ give constants; those in $u$ go like $(\ln t) / t$, so we finally have

$$
\begin{aligned}
\mathscr{N}(s, t) & \rightarrow-\frac{1}{4 \pi^{2}} g^{2} \gamma^{2} \\
& \times\left\{\frac{1}{[s(s-4)]^{1 / 2}} \ln \frac{(s-4)^{1 / 2}+s^{1 / 2}}{(s-4)^{1 / 2}-s^{1 / 2}}\right\} \ln (-t),
\end{aligned}
$$

where $\mathfrak{T}$ is the invariant Feynman amplitude for meson-nucleon scattering, with the entire result coming from Fig. 1 (a).

In lowest order, $\mathfrak{T}$ was given by

$$
\mathfrak{N}=-\frac{g^{2}}{s-1}-\frac{g^{2}}{u-1} \rightarrow-\frac{g^{2}}{s-1} \text { as } t \rightarrow \infty,
$$

so that altogether

$$
\begin{aligned}
& \mathscr{T}(s, t) \rightarrow-\frac{g^{2}}{s-1}- \frac{1}{4 \pi^{2}} g^{2} \gamma^{2} \frac{1}{[s(s-4)]^{1 / 2}} \\
& \times \ln \left[\frac{(s-4)^{1 / 2}+s^{1 / 2}}{(s-4)^{1 / 2}-s^{1 / 2}}\right] \ln (-t) .
\end{aligned}
$$

${ }^{2}$ R. E. Cutkosky, J. Math. Phys. 1, 429 (1960).
Now if $\mathscr{T}$ were to have a Regge-like behavior for large $t$, with a trajectory corresponding to a nucleon Regge pole in the $s$ channel, we should expect

$\operatorname{Ir}(s, t) \rightarrow($ function of $s) \times(-t)^{\alpha(s)}$ as $t \rightarrow \infty$,

where $\alpha(1)=0$. Comparing (3.4) and (3.3), it is clear that if the Reggeism actually obtains, the trajectory must be given by

$$
\alpha(s)=\frac{\gamma^{2}}{4 \pi^{2}} \frac{s-1}{[s(s-4)]^{1 / 2}} \ln \left[\frac{(s-4)^{1 / 2}+s^{1 / 2}}{(s-4)^{1 / 2}-s^{1 / 2}}\right] .
$$

The result $\alpha(1)=0$ is thus automatically ensured, and comes simply from the fact that the first term in $\mathscr{N}$ has a pole at $s=1$. Evidently, then, fourth order cannot really tell us anything and we must look further.

Further means sixth order, and it will become clear later, if it is not already obvious, that to compute sixthorder meson-nucleon scattering we need to know something about fourth-order photoproduction. The diagrams for this are the same as those shown in Figs. 1 and 2, with the outgoing meson replaced by a vector meson, and with the addition of the diagrams shown in Fig. 3. These diagrams are possible because of the presence of the "seagull picture," in which two vector mesons and two nucleon lines interact at a point, which results from an $A^{2} \phi^{\dagger} \phi$ term in the Lagrangian for a scalar-vector interaction, and which is necessary for the gauge invariance and renormalizability of the theory. It will subsequently be seen to be the origin of the basic difference in Regge behavior of the scalar and spinor nucleon theories.

The photoproduction covariant amplitude is a vector, which involves three scalar functions, among which current conservation provides one relation. It is again straightforward to calculate the double spectral functions; the analog of Fig. 1(a) gives

$$
\begin{aligned}
\rho_{\mu}{ }^{13}(s, t)= & (3-2 t) \rho_{0}(s, t) \\
& \times\left[\left(\frac{s-2}{4-s-t}\right) p_{1 \mu}+p_{2 \mu}+\left(\frac{2-s}{4-s-t}\right) q_{2 \mu}\right]
\end{aligned}
$$

where $q_{2 \mu}$ is the outgoing vector meson momentum. $\rho_{0}$ is
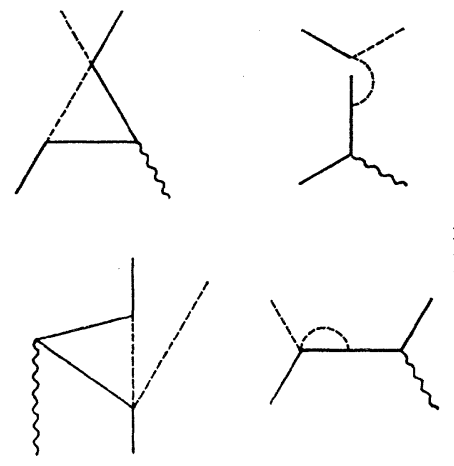

FIg. 3. Additional graphs for fourth-order photoproduction. 
Frg. 4. The sequence of box diagrams.
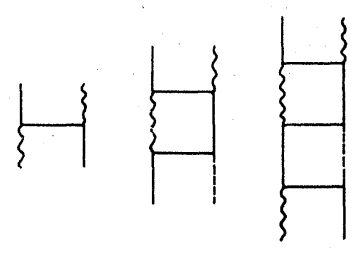

For large $t, I_{0}(-t, t)$ behaves like $\ln t / t^{2}$, while $I_{0}(s, t)$ is like $1 / t$; in both the amplitudes multiplied by $p_{1 \mu}$ and $p_{2 \mu}$, the terms proportional to $I_{0}(s, t)$, which are simply analogous to the meson scattering results, are thus swamped by unpleasant $s$-independent pieces. However, in the total $I_{\mu}(s, t)$, given by the sum of (3.7) and (3.8), all this stuff cancels out leaving just

$$
I_{\mu}(s, t) \rightarrow-2 t\left\{[(2-s) / t] I_{0}(s, t) p_{1 \mu}+I_{0}(s, t) p_{2 \mu}\right\} .
$$

defined in Eq. (3.1). The crossed box, analogous to Fig. 1(b), is obtained from (3.6) by setting $s \rightarrow u$, $p_{1} \rightarrow p_{2}$ and changing the over-all sign.

Before commenting on the renormalization graphs, let us first discuss backing up to the actual amplitude from these double spectral functions. Evidently, we cannot do here as we could in the scattering, namely just take the asymptotic form in $t$ of $\rho_{\mu}{ }^{13}$ and then infer that of the total amplitude. If we let $t \rightarrow \infty$ in (3.6), then $(s-2) /(4-s-t) \rightarrow-(s-2) / t$, and we lose a power of convergence in $s$ which makes the dispersion integral

$$
\int \frac{\rho\left(s^{\prime}, t\right) d s^{\prime}}{s^{\prime}-s}
$$

not exist. What this effectively means is that, if we first evaluate the dispersion integral in $s$ and then take the limit $t \rightarrow \infty$, we must get an extra power of $\ln t$ in the result. Thus, the amplitude obtained from (3.6) will contain terms going like $(\ln t)^{2}$, which, if uncancelled, would destroy any possibility of Regge-like behavior. In fact, of course, the $(\ln t)^{2}$ terms are precisely cancelled by the crossed graph of Fig. 1(b), and the remaining $(\ln t)$ terms all come from Fig. 1(a). In effect, then, one may say that just as in the meson-nucleon scattering, the dominant terms at large $t$ in fourth-order photoproduction come from the uncrossed box.

To clarify this cancellation a bit more fully, we may actually carry out the dispersion integral in $s$ over (3.6) and obtain the total amplitude; we find from (3.6) that

$$
\begin{aligned}
I_{\mu}(s, t)= & (3-2 t)\left\{\left[\frac{s-2}{4-s-t} I_{0}(s, t)\right.\right. \\
& \left.\left.-\frac{2-t}{4-s-t} I_{0}(4-t, t)\right] p_{1 \mu}+\left[I_{0}(s, t)\right] p_{2 \mu}\right\} .
\end{aligned}
$$

Here, $I_{\mu}(s, t)$ denotes the imaginary part in $t$ of the complete amplitude, and $I_{0}$ is simply the $s$ dispersion integral of $\rho_{0}$. From the crossed graph, on the other hand, we obtain a contribution

$$
\begin{aligned}
& I_{\mu}(s, t)=(3-2 t)\left\{\left[I_{0}(4-s-t, t)\right] p_{1 \mu}\right. \\
& \left.\quad+\left[\frac{s+t-2}{s} I(4-s-t, t)-\frac{t-2}{s} I(4-t, t)\right] p_{2 \mu}\right\} .
\end{aligned}
$$

Comparing this with (3.6), the prescription is clear; just as in the meson- $N$ case, set $t \rightarrow \infty$ in the uncrossed box and ignore the crossed box, and treat all the spin coefficients of $\rho_{0}(s, t)$ as if they were constant multiples.

The cancellation we have just described (which we will call the " $\log ^{2}$ " cancellation, from now on, just to give it a name) has analogs in all processes involving external vector mesons, and it keeps recurring in each order of perturbation theory. In all the cases we have checked, the cancellation in fact occurs in just the same way as outlined here. Yet we cannot prove that the cancellation must occur in all orders; as is seen later, we are able to calculate just the asymptotic form of the double spectral functions, for large $t$, in all orders, and with only this information we cannot construct the amplitude itself without assuming that the " $\log ^{2}$ " cancellation occurs. In our earlier treatment in the $J$ plane, the problem of the " $\log ^{2}$ " cancellation is buried in the question of subtractions in the partial wave dispersion relations.

\section{SIXTH-ORDER MESON-NUCLEON SCATTERING}

Having derived the formulas (3.7), (3.8), and (3.9), and having computed the various fourth-order photoproduction renormalization graphs, we are now in a position to tackle sixth-order meson- $N$ scattering. Not surprisingly, there are a multitude of graphs in sixth order, and among these we must seek some sort of simplification. As a guide, let us recall the situation in meson- $N$ scattering without virtual vector mesons. There, the leading term in $t$, in each order of perturbation theory, comes only from two-particle cuts in $s$. The important set of pictures, ${ }^{3}$ in fact, is just the set of boxes indicated in Fig. 4. We may hope the same situation obtains in our problem, involving virtual vector instead of virtual scalar mesons. To check this point, we have explicitly computed all the diagrams with two-particle cuts in sixth order, as well as some of those with threeparticle cuts. Let us briefly indicate how this was done.

To include all two-particle cuts, we must find all the diagrams contributing to the double spectral functions $\rho_{13}(s, t)$ and $\rho_{12}(s, u)$, with thresholds at $s=4$. For the first of these, the diagrams are shown in Fig. 5(a) to (f). Analytically, we may write, using the Cutkosky rules, ${ }^{2}$ the sixth-order double spectral function as follows, with

3 J. C. Polkinghorne, J. Math. Phys. (to be published); P. Federbush and M. Grisaru, Ann. Phys. (N. Y.) 22, 263, 299 (1963); G. Tiktopoulos, Phys. Rev. (to be published). 


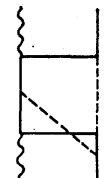

(a)

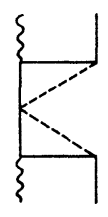

(e)

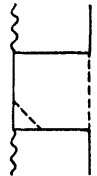

(b)

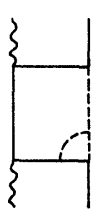

(f)

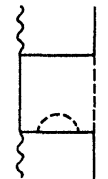

(c)

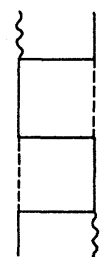

(g)

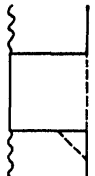

(d)

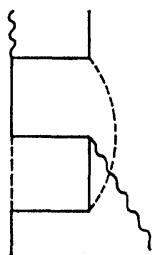

(h)
FIG. 5. Sixth-order meson-nucleon scattering graphs.

$p=p_{1}+q_{1}:$

$$
\begin{aligned}
\rho_{13}(s, t) & =-\frac{1}{2} \cdot \frac{g \gamma}{2 \pi} \int d^{4} q \delta\left(q^{2}+1\right) \delta\left[\left(p_{2}-q\right)^{2}+1\right] \\
\times \delta & {\left[(p-q)^{2}+1\right]\left(2 p_{2}-q\right)_{\mu} J_{\mu}\left[s,-\left(q-p_{1}\right)^{2}\right] . }
\end{aligned}
$$

Here, $J_{\mu}(s, u)$ is the imaginary part in $u$ of the fourthorder photoproduction; Eq. (4.1) thus contains the contributions of two-particle cuts in Figs. 5(a) through (f). We may insert in $(4.1)^{4} 1=2 \int d \theta^{2} \delta_{p}\left(\left(q-p_{1}\right)^{2}+\theta^{2}\right)$. Then, defining

$$
\begin{aligned}
K\left(s, t ; \theta^{2}\right)=- & \frac{g \gamma}{2 \pi} \int d^{4} q \delta\left(q^{2}+1\right) \delta\left[\left(p_{2}-q\right)^{2}+1\right] \\
& \times \delta\left[(p-q)^{2}+1\right] \delta_{p}\left[\left(q-p_{1}\right)^{2}+\theta^{2}\right],
\end{aligned}
$$

we have

$$
\rho_{13}(s, t)=\int d \theta^{2} K\left(s, t ; \theta^{2}\right)\left\langle\left(2 p_{2}-q\right)_{\mu} J_{\mu}\left(s, \theta^{2}\right)\right\rangle,
$$

where the symbol \langle\rangle means that the vectors $q_{\mu}$ appearing inside it are to be replaced by their "averages" calculated from the four-dimensional integral in (4.2). Explicitly, using the breakup indicated in Sec. 3 for the fourth-order photoproduction, and writing

$$
\begin{gathered}
J_{\mu}\left[s,-\left(p_{1}-q\right)^{2}\right]=J_{1}\left[s,-\left(p_{1}-q\right)^{2}\right] p_{1 \mu} \\
+J_{2}\left[s,-\left(p_{1}-q\right)^{2}\right](p-q)_{\mu} \\
+J_{3}\left[s,-\left(p_{1}-q\right)^{2}\right] q_{\mu},
\end{gathered}
$$

we have

$$
\begin{aligned}
\rho_{13}(s, t)=\int d \theta^{2} K\left(s, t ; \theta^{2}\right) \\
\\
\quad \times\left[\frac{1}{2} s J_{2}\left(s, \theta^{2}\right)+\left(1-t+\frac{1}{2} \theta^{2}\right) J_{1}\left(s, \theta^{2}\right)\right] .
\end{aligned}
$$

Note that if $\theta^{2}=1$, the kernel $K$ is essentially just the "spinless box" weight function denoted previously by $\rho_{0}(s, t) \cdot \theta^{2}$ itself is the crossed momentum transfer in the fourth-order photoproduction amplitude.

${ }^{4}$ Here, $\delta_{p}$ means the positive energy part of the delta function.
It is not hard to convince oneself by studying the properties of $K$ and Eq. (4.5), that to extract $\rho_{13}(s, t)$ at large $t$, we need $J_{1}$ and $J_{2}$ at large $\theta^{2}$. We must therefore look back at our calculation of fourth-order photoproduction, and at the absorptive parts in, and for large values of, the crossed momentum transfer. This is easily done in principle, though somewhat messy algebraically in practice.

To evaluate $J_{1}$ and $J_{2}$ in fourth order, we must compute the six graphs in Fig. 6(a)-(f). The last five of these are functions only of $u$; they would be represented by single integral terms in a Mandelstam representation for the photoproduction amplitude. Their computation is straightforward; we may choose just one, say Fig. 6(c), to illustrate how it is carried out.

The imaginary part of Fig. 6(c), cut across the bubble, is given, according to the Cutkosky rule, by ${ }^{5}$

$$
\begin{aligned}
-\frac{1}{2}\left(\frac{1}{2 \pi}\right)^{2} \int d^{4} k\left(2 p_{1}-q\right)_{\mu}\left(\frac{1}{\theta^{2}-1}\right)^{2} \delta\left[(R-k)^{2}+1\right] \\
\times \delta\left(k^{2}+1\right)(2 R-k)_{\lambda}\left(\delta_{\lambda \sigma}+k_{\lambda} k_{\sigma}\right)(2 R-k)_{\sigma}
\end{aligned}
$$

where the momenta are labeled as shown in the figure, and $R^{2}=-\left(p_{1}-q\right)^{2}=\theta^{2}$. We may simplify this to

$$
\begin{aligned}
-\frac{1}{2}\left(\frac{1}{2 \pi}\right)^{2}\left(2 p_{1}-q\right)_{\mu}\left(\frac{1}{\theta^{2}-1}\right)^{2} & \left(4 \theta^{2}-\theta^{4}\right) \\
& \times \int d^{4} k\left(2 R \cdot k+\theta^{2}\right) \delta\left(k^{2}+1\right) .
\end{aligned}
$$

The integral is easily evaluated and equals just

$$
\frac{\pi}{2}\left[\left(\theta^{2}-4\right) / \theta^{2}\right]^{1 / 2} \text {. }
$$

Altogether, then, we get

$$
\left\{-\frac{1}{2}\left(\frac{1}{2 \pi}\right)^{2} \frac{\pi}{2}\left(\frac{\theta^{2}-4}{\theta^{2}}\right)^{1 / 2} \frac{4 \theta^{2}-\theta^{4}}{\left(\theta^{2}-1\right)^{2}}\right\}\left(2 p_{1}-q\right)_{\mu},
$$

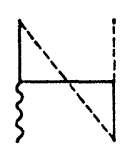

(0)

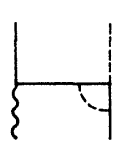

(d)

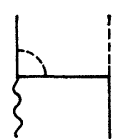

(b)

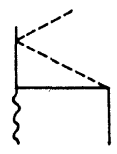

(e)

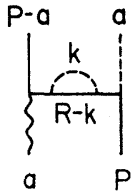

(c)

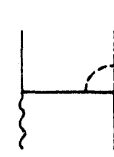

(f) $\sigma$ We choose to use the propagator $\left(\delta_{\mu \nu}+k_{\mu} k_{\nu}\right) /\left(k^{2}+1\right)$ for the vector meson here (though, as is well known, the extra $k_{\mu} k_{\nu}$ term is unnecessary if it is consistently ignored) because a slight simplification of the algebra occurs since $k_{\mu}\left(\delta_{\mu \nu}+k_{\mu} k_{\nu}\right)=0$. 
which, it may be noted, contributes entirely to $J_{1}$ and $J_{3}$ and not to $J_{2}$ at all.

The other graphs, Figs. 6(b), (d), (e), and (f), may be calculated in a similar manner. None of them contributes to $J_{2}$ either.

From Fig. 6(b), we get

$\left\{-\frac{1}{2}\left(\frac{1}{2 \pi}\right)^{2} \frac{\pi}{2}\left(\frac{\theta^{2}-4}{\theta^{2}}\right)^{1 / 2} \frac{\theta^{2}}{\theta^{2}-1}\right.$

$$
\left.\times\left[1-\frac{1}{\theta^{2}-4} \ln \left(\theta^{2}-3\right)\right]\right\}\left(2 p_{1}-q\right)_{\mu} ;
$$

from Fig. 6(a),

$$
\begin{aligned}
\left\{-\frac{1}{2}\left(\frac{1}{2 \pi}\right)^{2}\right. & \frac{\pi}{2}\left(\frac{\theta^{2}-4}{\theta^{2}}\right)^{1 / 2} \frac{\theta^{2}}{\theta^{2}-1} \\
& \left.\times\left[\frac{1}{2}-\frac{1}{\theta^{2}-4}+\frac{1}{\left(\theta^{2}-4\right)^{2}} \ln \left(\theta^{2}-3\right)\right]\right\}\left(2 p_{1}-q\right)_{\mu} ;
\end{aligned}
$$

from Fig. 6(e),

$$
\begin{aligned}
& \left\{-\frac{1}{2}\left(\frac{1}{2 \pi}\right)^{2} \frac{\pi}{2}\left(\frac{\theta^{2}-4}{\theta^{2}}\right)^{1 / 2}\right. \\
& \left.\quad \times\left[-\frac{1}{2}-\frac{1}{\theta^{2}-4}-\frac{1}{\left(\theta^{2}-4\right)^{2}} \ln \left(\theta^{2}-3\right)\right]\right\}\left(2 p_{1}-q\right)_{\mu} ;
\end{aligned}
$$

and finally from Fig. 6(f),

$$
\left\{-\frac{1}{2}\left(\frac{1}{2 \pi}\right)^{2} \frac{\pi}{2}\left(\frac{\theta^{2}-4}{\theta^{2}}\right)^{1 / 2}\left[\frac{1}{2}-\frac{3}{2\left(\theta^{2}-4\right)}\right]\right\}\left(2 p_{1}-q\right)_{\mu} .
$$

Asymptotically, for large $\theta^{2}$, these five amplitudes approach common factors times

$$
\begin{aligned}
& \left(-1+\frac{2}{\theta^{2}}+\cdots\right), \\
& \left(1+\frac{1}{\theta^{2}}-\frac{1}{\theta^{2}} \ln \theta^{2}+\cdots\right), \\
& \left(\frac{1}{2}-\frac{1}{2 \theta^{2}}+\frac{1}{\theta^{4}} \ln \theta^{2}+\cdots\right), \\
& \left(-\frac{1}{2}-\frac{1}{\theta^{2}}-\frac{1}{\theta^{4}} \ln \theta^{2}+\cdots\right), \\
& \left(\frac{1}{2}-\frac{3}{2 \theta^{2}}+\cdots\right) .
\end{aligned}
$$

The contribution to $J$ from the crossed box, Fig. 6(a), is not quite so easy. From Sec. 1 we already know the weight functions $\rho_{1}(u, t)$ and $\rho_{2}(u, t)$. $J_{1}$ and $J_{2}$ may be obtained from these with a dispersion integral over $t$. Unfortunately, in $J_{1}$ this integral needs a subtraction, so we must evaluate the subtraction constant by other means. For $t=0$, it is not too difficult to calculate $J_{1}$ directly from the box graph cut only in $u$, so it is convenient to make the subtraction at $t=0$.

In this way we find that for large $\theta^{2}, J_{1}$ approaches

$$
-\frac{1}{2}\left(\frac{1}{2 \pi}\right)^{2} \frac{\pi}{2}\left(\frac{\theta^{2}-4}{\theta^{2}}\right)^{1 / 2}\left[-\frac{1}{2}+\frac{1}{\theta^{2}} \ln \theta^{2}+O\left(\frac{1}{\theta^{4}}\right)\right]
$$

and $J_{2}$ behaves like

$$
-\frac{1}{2}\left(\frac{1}{2 \pi}\right)^{2} \frac{\pi}{2}\left(\frac{\theta^{2}-4}{\theta^{2}}\right)^{1 / 2}\left(\frac{1}{\theta^{2}} \ln \theta^{2}\right) .
$$

Combining this asymptotic form for the part of $J_{1}$ coming from Fig. 6(a) with the parts given earlier from Figs. 6(b) through 6(f), we find altogether that

$$
J_{1} \rightarrow-\frac{1}{2}\left(\frac{1}{2 \pi}\right)^{2} \frac{\pi}{2}\left(\frac{\theta^{2}-4}{\theta^{2}}\right)^{1 / 2}\left[O\left(\frac{1}{\theta^{4}} \ln \theta^{2}\right)\right] .
$$

Thus it turns out that, while the contribution of some of the individual graphs in Fig. 6(a) to (f) may even approach constants as $\theta^{2} \rightarrow \infty$, there is a remarkable amount of cancellation among them and the resultant $J_{1}$ and $J_{2}$ behave no worse than $\ln \theta^{2} / \theta^{4}$ and $\ln \theta^{2} / \theta^{2}$, respectively. As a consequence, $\rho_{13}(s, t)$, from Eq. (4.5), can behave no worse then $(\ln t)^{2} / t$ for large $t$. This will give rise to an amplitude which vanishes as $t \rightarrow \infty$ and which cannot therefore be important if the conjectured Regge-like behavior is correct.

This is the second of the apparently miraculous cancellations which perturbation theory affords. We return to a fuller discussion of it later.

To continue our hunt for the dominant terms in sixth order, we turn next to $\rho_{12}(s, u)$, which contains the contributions of Figs. $5(\mathrm{~g})$ and $(\mathrm{h})$. Figure $5(\mathrm{~g})$, it. can be observed, is the graph which in the totally spinless case provided the leading term. Thus far, then, we are supported in the view that the sequence of graphs of interest will be the same as in the spinless case. If this is indeed the case, the dominant spectral function alternates order by order between $\rho_{13}(s, t)$ and $\rho_{12}(s, u)$. The series we can expect is then not

$$
\begin{array}{r}
{\left[g^{2} /(s-1)\right]\left[1+\alpha \ln (-t)+\frac{1}{2} \alpha^{2} \ln ^{2}(-t)+\cdots\right]} \\
=\left[g^{2} /(s-1)\right](-t)^{\alpha(s)},
\end{array}
$$

but instead

$$
\begin{array}{r}
{\left[g^{2} /(s-1)\right]\left[1+\alpha \ln (-t)+\frac{1}{2} \alpha^{2} \ln ^{2} t+\frac{1}{6} \alpha^{3} \ln ^{3}(-t)+\cdots\right]} \\
=\left(g^{2} / s-1\right) \frac{1}{2}\left\{\left[(-t)^{\alpha}+(t)^{\alpha}\right]-\left[(-t)^{-\alpha}-t^{-\alpha}\right]\right\}
\end{array}
$$

just as in the spinor case. If the conjecture turns out to be verified, then, we will find "two equal and opposite Regge poles of opposite signatures. The even signature one is presumably the one to be associated with the nucleon, in accord with its zero spin. 
By the same argument that led to (4.5), we easily find

$$
\begin{aligned}
\rho_{12}(s, u) & =\int d \theta^{2} K\left(s, u ; \theta^{2}\right) \\
\times & {\left[I_{1}\left(s, \theta^{2}\right)\left(-1+u+\frac{s-\theta^{2}}{2}\right)+I_{2}\left(s, \theta^{2}\right)\left(\frac{s}{2}\right)\right] . }
\end{aligned}
$$

Here, $I_{1}$ and $I_{2}$ are the coefficients of $p_{1 \mu}$ and $p_{2 \mu}$ in the absorptive part in $t$ of the fourth-order photoproduction; evidently, they can be read off from Eqs. (3.7) and (3.8). Again we are interested in the large $\theta^{2}$ region, $\left(\theta^{2}\right.$ now is the ordinary, not the crossed, momentum transfer in fourth-order photoproduction) and this is given in (3.9). Figure 5(b) then serves the purpose only of removing a $(\ln t)^{2}$ term from Fig. $5(\mathrm{~g})$, as explained in Sec. 3.

The resulting expression for the weight function for large $u$ is

$$
\begin{aligned}
& \rho_{12}(s, u) \rightarrow \int d \theta^{2} K\left(s, u ; \theta^{2}\right) \\
& \times\left\{\left(\theta^{2}-2 u\right)(2-s)-s \theta^{2}\right\} I_{0}\left(s, \theta^{2}\right) .
\end{aligned}
$$

Here, to be specific,

$$
\begin{aligned}
& K=\left(\frac{g \gamma}{2 \pi}\right) \frac{1}{2[s(s-4)]^{1 / 2}} \frac{1}{\left[\left(\theta^{2}-\beta_{-}\right)\left(\theta^{2}-\beta_{+}\right)\right]^{1 / 2}}, \\
& \beta_{+}>\beta_{-} ;
\end{aligned}
$$

the range of integration is $1 \leq \theta^{2} \leq \beta_{-}$. Finally,

$$
I_{0}\left(s, \theta^{2}\right)=-\frac{\gamma^{3} g}{4 \pi \theta^{2}} \frac{1}{[s(s-4)]^{1 / 2}} \ln \frac{(s-4)^{1 / 2}+s^{1 / 2}}{(s-4)^{1 / 2}-s^{1 / 2}},
$$

when $\theta^{2} \gg s$.

For large $u, \beta_{-}$is proportional to $u$. The integral is not difficult to evaluate; for later reference it is useful to note that when $u$ is large, $u$ itself is more effective in the curly bracket of Eq. (4.7) than $\theta^{2}$. Explicitly,

$$
\begin{aligned}
& \int d \theta^{2} K I_{0} \sim \ln u / u \text { for large } u, \\
& \int d \theta^{2} K \theta^{2} I_{0} \sim \text { constant. }
\end{aligned}
$$

In the end, then, we have

$$
\rho_{12}(s, u) \rightarrow-2(2-s) u \int d \theta^{2} K\left(s, u ; \theta^{2}\right) I_{0}\left(s, \theta^{2}\right) .
$$

It is worth remarking also, incidently, that $\int d \theta^{2} K I_{0}$ is precisely what one would obtain in the sixth order of a completely spinless problem; the net effect of making the intermediate mesons vector is then simply to multiply the spinless sixth-order result by $-2(2-s) u$.
The final result is

$$
\rho_{12}(s, u) \rightarrow-\frac{g^{2} \gamma^{4}}{16 \pi^{2}} \frac{\left(1-\frac{1}{2} s\right)}{s(s-4)} \ln \frac{(s-4)^{1 / 2}+s^{1 / 2}}{(s-4)^{1 / 2}-s^{1 / 2}} \ln t .
$$

Even without further thought, it is clear that the conjectured Regge behavior fails, since the sixth order is not proportional to $s-1$.

Had the Regge behavior been correct, on the basis of the second- and fourth-order calculations, we should have found in sixth order that

$$
\begin{aligned}
\mathscr{T}(s, t) & \rightarrow-\frac{g^{2}}{s-1} \frac{[\alpha(s)]^{2}}{2}(\ln t)^{2} \\
& =-\frac{g^{2} \gamma^{4}}{32 \pi^{4}} \frac{s-1}{s(s-4)}\left[\ln \frac{(s-4)^{1 / 2}+s^{1 / 2}}{(s-4)^{1 / 2}-s^{1 / 2}}\right]^{2}(\ln t)^{2} .
\end{aligned}
$$

The weight function which corresponds to this, and which we therefore should have found, is

$$
\rho_{12}(s, u) \rightarrow-\frac{g^{2} \gamma^{4}}{8 \pi^{2}} \frac{s-1}{s(s-4)} \ln \frac{(s-4)^{1 / 2}+s^{1 / 2}}{(s-4)^{1 / 2}-s^{1 / 2}} \ln t .
$$

Comparing with (4.11), what we actually found was the desired result but with $s-1$ replaced by $(s-2) / 4$.

To back up from the weight function in (4.11) to the sixth-order scattering amplitude is straightforward here, in that $\rho$ behaves like $\ln s / s$ for large $s$, so that an unsubtracted integral in $s$ exists. There is nothing here like the difficulty in fourth-order photoproduction, which was cured by the "log2" cancellation.

It is obvious that inclusion of multiparticle cuts cannot alter the failure of the conjecture, since the sixthorder amplitude it predicts, in fact, has a two-particle threshold in $s$. It is nevertheless interesting to ask if the amplitude in (4.11) is the dominant one. We have not exhaustively studied all three-particle cuts, but the ones that we have investigated behave at worst like $\ln t$ in the amplitude, and are thus dominated by (4.11).

\section{ASYMPTOTIC UNITARITY: SCALAR-VECTOR COMPTON SCATTERING}

In spite of the fact that the calculation of the sixthorder meson- $N$ scattering amplitude, described in the previous section, makes it clear that the conjecture of Regge-like behavior for the "elementary" nucleon fails for spin zero nucleons, it may be of interest to try to pin down the origin of the failure more precisely. We do this next by studying the asymptotic behavior in $t$ of the general term in the perturbation series.

Once we begin to study higher order terms, it is clear that we should no longer continue to concentrate on meson-nucleon scattering, but should instead focus on "Compton" scattering, that is, vector meson-scalar nucleon scattering. If it is correct to keep only twoparticle intermediate states in looking for the leading 
terms at large $t$, then the unitarity relations express meson-nucleon scattering in terms of photoproduction, and photoproduction in terms of itself and Compton scattering. Only Compton scattering couples purely to itself through two-particle unitarity, and it is therefore, in some sense, more fundamental, in that it must be understood first, before it is possible to calculate the behavior of the other two processes. Nevertheless, even though from now on we limit ourselves to Compton scattering, the fourth- and sixth-order meson-nucleon results in Secs. 3 and 4 will be quite useful as guides to what we may expect.

We shall find it very convenient to compare many of our results to the totally spinless problem, in other words, to "Compton scattering" of scalar mesons from scalar nucleons, and a brief discussion of this process first may not be out of place.

The scalar meson scalar nucleon scattering amplitude will be denoted by $\Re(s, t) ; I(s, t)$ will be the absorptive part in $t$ and $J(s, u)$ the absorptive part in $u$. Thus, if $\mathfrak{T}(s, t)$ satisfies the Mandelstam representation, so that

$$
\begin{aligned}
\mathscr{T}(s, t)= & -\frac{g^{2}}{s-1}-\frac{g^{2}}{u-1}+\frac{1}{\pi} \int \frac{f_{1}\left(s^{\prime}\right)}{s^{\prime}-s} d s^{\prime}+\frac{1}{\pi} \int \frac{f_{2}\left(u^{\prime}\right)}{u^{\prime}-u} d u^{\prime} \\
& +\frac{1}{\pi} \int \frac{f_{3}\left(t^{\prime}\right)}{t^{\prime}-t} d t^{\prime}+\frac{1}{\pi^{2}} \iint \frac{\rho_{13}\left(s^{\prime}, t^{\prime}\right)}{\left(s^{\prime}-s\right)\left(t^{\prime}-t\right)} d s^{\prime} d t^{\prime} \\
& +\frac{1}{\pi^{2}} \iint \frac{\rho_{23}\left(u^{\prime}, t^{\prime}\right)}{\left(u^{\prime}-u\right)\left(t^{\prime}-t\right)} d u^{\prime} d t^{\prime} \\
& +\frac{1}{\pi^{2}} \iint \frac{\rho_{12}\left(s^{\prime}, u^{\prime}\right)}{\left(s^{\prime}-s\right)\left(u^{\prime}-u\right)} d s^{\prime} d u^{\prime},
\end{aligned}
$$

where

and

$$
f_{1}(s)=f_{2}(s), \quad \rho_{13}(s, t)=\rho_{23}(s, t),
$$

$$
\rho_{12}(s, u)=\rho_{12}(u, s),
$$

by crossing symmetry, we may write

$$
I(s, t)=f_{3}(t)+\frac{1}{\pi} \int \frac{\rho_{13}\left(s^{\prime}, t\right)}{s^{\prime}-s} d s^{\prime}+\frac{1}{\pi} \int \frac{\rho_{23}\left(u^{\prime}, t\right)}{u^{\prime}-u} d u^{\prime}
$$

and

$$
\begin{aligned}
& J(s, u)=-\pi g^{2} \delta(u-1)+f_{2}(u) \\
& +\frac{1}{\pi} \int \frac{\rho_{23}\left(, u t^{\prime}\right)}{t^{\prime}-t} d t^{\prime}+\int \frac{\rho_{12}\left(s^{\prime}, u\right)}{s^{\prime}-s} d s^{\prime} .
\end{aligned}
$$

Fig. 7. Two-particle cuts contributing to $\rho(s, t)$.
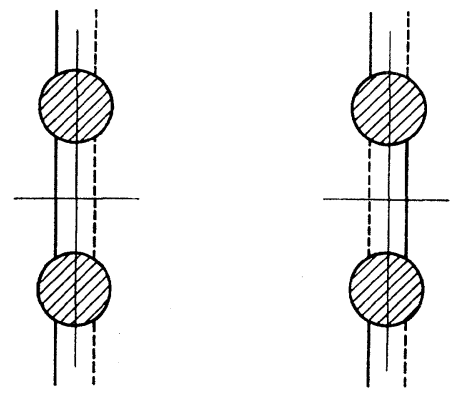

Note that $I$ and $J$ are not related in any particular way.

If (5.1) is in fact correct, then when $t \rightarrow \infty$ for fixed $s$, which is the limit we concern ourselves with, we clearly find

$$
\mathfrak{M}(s, t) \rightarrow-\frac{g^{2}}{s-1}+\frac{1}{\pi} \int \frac{f_{1}\left(s^{\prime}\right)}{s^{\prime}-s} d s^{\prime} .
$$

This is manifestly not a Regge-like asymptotic form; it corresponds instead to an energy-independent $\delta_{l 0}$ term in the angular momentum plane. It also contains the nucleon pole term at $s=1$ and thus fits perfectly with the idea that an elementary nucleon, such as we are presumably dealing with here, is not a Regge pole.

We may nevertheless continue to look for Regge-like behavior, though it evidently cannot be Regge-like behavior connected with the existence of a particular particle like the nucleon. To do this we must look, not at the leading terms in $t$ in each order of perturbation theory, as these give (5.4), but at something smaller. It is well known what kind of Feynman diagrams will give Reggeism; namely, the ladder graphs shown in Fig. 4. It is furthermore well known that only the two-particle cuts in these diagrams produce the dominant terms. For our dispersion approach, then, we need to write down two-particle unitarity in the $s$ channel. If we write down the contribution of dispersion graphs like Fig. 7 to the weight function $\rho_{13}(s, t)$, we have

$$
\begin{aligned}
\rho_{13}(s, t)=\int d t_{1} \int d t_{2} K\left(s, t, t_{1}, t_{2}\right) \\
\\
\times\left\{I\left(s, t_{1}\right) * I\left(s, t_{2}\right)+J\left(s, t_{1}\right)^{*} J\left(s, t_{2}\right)\right\} .
\end{aligned}
$$

This is trivially derived as outlined in the sixth-order example in Sec. 4. The function $K$ is given by

$$
K=-\frac{1}{8 \pi^{2}} \frac{1}{[s(s-4)]^{1 / 2}} \frac{1}{\left\{t^{2}+t_{1}^{2}+t_{2}{ }^{2}-2 t t_{1}-2 t t_{2}-2 t_{1} t_{2}-\left[4 t t_{1} t_{2} /(s-4)\right]\right\}^{1 / 2}} .
$$

The integration in the $t_{1}, t_{2}$ plane is over the region where the argument of the square root is positive, and where the functions $I$ and $J$ do not vanish. Thus the region is

$$
\begin{gathered}
t_{1} \geq 1, \quad t_{2} \geq 1 \\
t \geq t_{1}+t_{2}+\frac{2 t_{1} t_{2}}{s-4}+2\left(t_{1} t_{2}\right)^{1 / 2}\left(1+\frac{t_{1}}{s-4}\right)^{1 / 2}\left(1+\frac{t_{2}}{s-4}\right)^{1 / 2}
\end{gathered}
$$



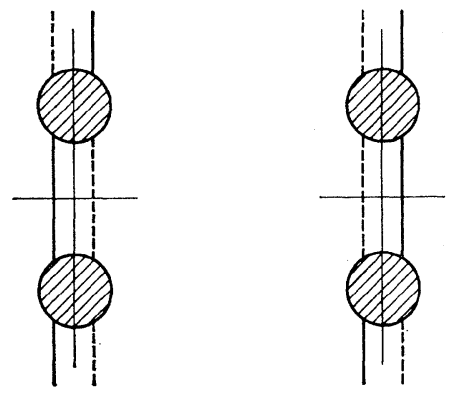

Fig. 8. Two-particle cuts contributing to $\rho(s, u)$.

The double spectral function $\rho_{12}(s, u)$, as indicated in Fig. 8, obviously satisfies an analogous equation:

$$
\begin{aligned}
\rho_{12}(s, u)=\int d t_{1} \int d t_{2} K\left(s, u, t_{1}, t_{2}\right) \\
\\
\times\left\{I\left(s, t_{1}\right)^{*} J\left(s, t_{2}\right)+J\left(s, t_{1}\right)^{*} I\left(s, t_{2}\right)\right\} .
\end{aligned}
$$

If we expand $\rho, I$, and $J$ in a perturbation series, and write, for example,

$$
\rho_{13}(s, t)=\sum_{n=1}^{\infty} g^{2 n} \rho_{13}{ }^{(n)}(s, t)
$$

then we have from (5.5)

$$
\rho_{13}{ }^{(n)}(s, t)=\sum_{m=1}^{n-1} \int d t_{1} \int d t_{2} K\left\{I^{m *} I^{n-m}+J^{m *} J^{n-m}\right\} .
$$

Equation (5.8) may now be used to derive by induction the asymptotic form of the two-particle cuts. Suppose that a Regge-like asymptotic form is, in fact, correct for these graphs. Then we would expect to have

$$
\begin{aligned}
\mathscr{N}(s, t) \rightarrow\left(g^{2} / 2 t\right)\left[\left[(-t)^{g^{2} \alpha(s)}+t^{g^{2} \alpha(s)}\right]\right. \\
\left.-\left[(-t)^{-g^{2} \alpha(s)}-t^{-g^{2} \alpha(s)}\right]\right]
\end{aligned}
$$

as $t \rightarrow \infty$ for a fixed $s$. Expanding this in a perturbation series, we have

$$
\begin{array}{rlr}
\mathscr{T}^{(m)}(s, t) & \rightarrow \frac{1}{t} \frac{\alpha^{m-1}}{(m-1) !}[\ln (-t)]^{m-1}, & m \text { even } \\
& \rightarrow \frac{1}{t} \frac{\alpha^{m-1}}{(m-1) !}(\ln t)^{m-1}, \quad m \text { odd } .
\end{array}
$$

From this we can get the forms to be expected for $I$ and $J$ :

$$
\begin{aligned}
I^{(m)} & \rightarrow-\frac{\pi}{t} \frac{\alpha^{m-1}}{(m-2) !}[\ln (-t)]^{m-2}, & & m \text { even }, \\
& \rightarrow 0, & & m \text { odd } \\
J^{(m)} & \rightarrow 0, & & m \text { even }, \\
& \rightarrow-\frac{\pi}{t} \frac{\alpha^{m-1}}{(m-2) !}(\ln t)^{m-2}, & & m \text { odd } .
\end{aligned}
$$

The induction now proceeds as follows. Suppose we assume Eqs. (5.10), (5.11), and (5.12) for all $m \leq n-1$. Then we know what to put into the right-hand side of (5.6) and (5.8), and we may therefore compute the spectral function in $n$th order, and then evaluate $I, J$, and $\mathscr{T}$ in $n$th order by integrating.

Before we actually do this, let us notice that the alternation between $-t$ and $t$ in even and odd orders comes out correctly. Suppose $n$ is even. Then in (5.6), if $m$ is even, then $n-m$ is also even, and there are nonzero contributions from the $I^{m} I^{n-m}$ term; if $m$ is odd, so is $n-m$, and we get something from the $J^{m} J^{n-m}$ term. Thus, $\rho_{13}^{(n)} \neq 0$. If $n$ is odd, on the other hand, $m$ even means $n-m$ odd and neither $I I$ nor $J J$ contributes; the same is true if $m$ is odd, for then $n-m$ is even. Thus, $\rho_{13}{ }^{(n)}=0$ for $n$ odd. The reverse is evidently true for $\rho_{12}{ }^{(n)}$, from (5.8).

Now let us take $n$ even. Then by explicitly using (5.11) and (5.12), we find from (5.6) that

$$
\begin{gathered}
\rho_{13}{ }^{(n)}(s, t)=\pi^{2} \sum_{m=1}^{n-1}\left(\alpha^{m-1}\right)^{*}\left(\alpha^{n-m-1}\right) \frac{1}{(m-2) !} \frac{1}{(n-m-2) !} \\
\times \int d t_{1} \int d t_{2} K\left(s, t, t_{1}, t_{2}\right) \frac{\left(\ln t_{1}\right)^{m-2}}{t_{1}} \frac{\left(\ln t_{2}\right)^{n-m-2}}{t_{2}} .
\end{gathered}
$$

The integral here can be computed for large $t$ (i.e., for $i \gg s)$ and we find

$$
\begin{array}{r}
\int d t_{1} \int d t_{2} K \frac{\left(\ln t_{1}\right)^{m-2}}{t_{1}} \frac{\left(\ln t_{2}\right)^{n-m-2}}{t_{2}} \rightarrow-\frac{1}{8 \pi^{2}} \frac{1}{[s(s-4)]^{1 / 2}} \\
\times \frac{(m-2) !(n-m-2) !}{(n-2) !} \frac{(\ln t)^{n-2}}{t} \cdot
\end{array}
$$

It is also easily seen that the dominant effect comes from $t_{1}, t_{2} \gg s$, confirming our use of the asymptotic values of $I$ and $J$. Inserting this into the expression for $\rho$, we obtain the asymptotic form:

$$
\begin{aligned}
& \rho_{13}{ }^{(n)}(s, t) \rightarrow-\frac{1}{8} \frac{1}{[s(s-4)]^{1 / 2}} \\
& \times \sum_{m=1}^{n-1} \frac{\left(\alpha^{m-1}\right)^{*}\left(\alpha^{n-m-1}\right)}{(n-2) !} \frac{(\ln t)^{n-2}}{t}, \\
&=-\frac{1}{8} \frac{1}{[s(s-4)]^{1 / 2}} \frac{1}{(n-2) !} \\
& \quad \times \frac{\alpha^{n-1}-\left(\alpha^{*}\right)^{n-1}}{\alpha-\alpha^{*}} \frac{(\ln t)^{n-2}}{t} .
\end{aligned}
$$

The expected form for $\rho_{13}{ }^{(n)}(s, t)$, if the induction were to work, would be

$\rho_{13}{ }^{(n)}(s, t) \rightarrow-(\pi / t)[1 /(n-2) !] \operatorname{Im}\left(\alpha^{n-1}\right)(\ln t)^{n-2}$ 
as can be seen from (5.11). Our result, (5.15), is precisely this provided we identify $\operatorname{Im} \alpha$ with $1 / 8 \pi[s(s-4)]^{1 / 2}$.

The Regge-type behavior has thus been proved to be correct, for two-particle cuts, and the value of $\alpha$ has been computed:

$$
\alpha=\frac{g^{2}}{8 \pi^{2}[s(s-4)]^{1 / 2}} \ln \frac{(s-4)^{1 / 2}+s^{1 / 2}}{(s-4)^{1 / 2}-s^{1 / 2}} .
$$

These results are, of course, all well known ${ }^{3}$; we have presented them here in such detail because the particular technique used in deriving them is different and it is precisely this technique which we use in solving the problem of real interest, to which we now turn.

The problem we wish to study is vector-meson scattering from scalar nucleons, and we want to look at the amplitude for this in the large $t$, fixed $s$ limit, to see if we find a Regge asymptotic behavior with a trajectory which can be associated with the nucleon. We would like to calculate the leading term for large $t$ in each order of perturbation theory and sum these terms, in the hope that this will give us the correct asymptotic form of the whole series. On the basis of our study of sixth-order meson-nucleon scattering with virtual vector mesons, we were encouraged to expect that the dominant terms come from two-particle cuts in the $s$ channel and we shall assume this is true.

The Compton scattering amplitude, which we denote $\mathfrak{T}_{\mu \nu}$ where $\nu$ and $\mu$ are the initial and final polarization directions, is a tensor containing four independent scalar amplitudes. Let us write

$$
\begin{aligned}
\mathfrak{T}_{\mu \nu}=4 A & P_{\nu} P_{\mu}+4 B R_{\nu} R_{\mu} \\
& +2 C\left(P_{\nu} R_{\mu}+R_{\nu} P_{\mu}\right)+D \delta_{\mu \nu}+E k_{2 \mu} k_{1 \nu} \\
& +2 F\left(k_{2 \mu} P_{\nu}+P_{\mu} k_{1 \nu}\right)+2 G\left(k_{2 \mu} R_{\nu}+R_{\mu} k_{1 \nu}\right),
\end{aligned}
$$

where $A$ through $G$ are scalar functions of $s, t$, and $u$. $P=p_{1}+k_{1}$ and $R=p_{1}-k_{2}$ if we call the initial and final nucleon and vector-meson four momenta $p_{1}, p_{2}, k_{1}$, and $k_{2}$. Then $-P^{2}=s$ and $-R^{2}=u$. Only four of the seven functions $A$ through $G$ actually count; current conservation (that is, the statement that $\mathfrak{M}_{\mu \nu} k_{\nu}=0$ ) gives three relations among them; for large $t$,

$$
\begin{aligned}
& C=-(1 / t)[2 s A+D+2 F], \\
& B=(1 / 2 t) D, \\
& G=(1 / t)[D-E-s F] .
\end{aligned}
$$

As we said earlier, we shall restrict ourselves to twoparticle states since, at least through sixth order, they provide the biggest contribution at large $t$. In analogy to (5.5), then, we may write

$$
\begin{aligned}
\rho_{13}(s, t)_{\mu \nu} & =-\int d t_{1} \int d t_{2} K\left(s, t, t_{1}, t_{2}\right) \\
& \times\left\langle I\left(s, t_{1}\right)_{\mu \lambda} * I\left(s, t_{2}\right)_{\lambda \nu}+J\left(s, t_{1}\right)_{\mu \lambda} * J\left(s, t_{2}\right)_{\lambda \nu}\right\rangle .
\end{aligned}
$$

The symbol \langle\rangle , as was mentioned in Sec. 4, implies that the intermediate four momenta in Fig. 8 are averaged as follows:

$$
\left\langle l_{\mu}\right\rangle=\frac{\int d^{4} l \delta\left(l^{2}+1\right) \delta\left[\left(p_{1}-l\right)^{2}+t_{1}\right] \delta\left[\left(p_{2}-l\right)^{2}+t_{2}\right] \delta\left[(P-l)^{2}+1\right] l_{\mu}}{\int d^{4} l \delta\left(l^{2}+1\right) \delta\left[\left(p_{1}-l\right)^{2}+t_{1}\right] \delta\left[\left(p_{2}-l\right)^{2}+t_{2}\right] \delta\left[(P-l)^{2}+1\right]},
$$

with an analogous expression for $\left\langle l_{\mu} l_{\nu}\right\rangle$.

We intend to use the same induction arguments used earlier in this section for the totally spinless case; the $t-u$ alternation in successive orders will then follow here as it did in that case. As an explicit verification of this fact, we may recall the second "incredible cancellation" in the sixth-order meson-nucleon case, in which the large terms of all the various graphs contributing to $\rho_{13}(s, t)$ in sixth order cancelled out. Once it is accepted that the alternation occurs, we need no longer distinguish between $I$ and $J$, as was evident in (5.13), so we may simplify (5.20) to

$$
\rho_{13}(s, t)_{\mu \nu}=-\int d t_{1} \int d t_{2} K\left\langle I^{*}\left(s, t_{2}\right)_{\mu \lambda} I\left(s, t_{1}\right)_{\lambda_{\nu}}\right\rangle .
$$

Before proceeding with the induction argument, we must reduce the spinology in (5.22) to the point that we get scalar relationships analogous to the completely spinless case. Once this is done, we should be able to just read off the results by analogy.

It is algebraically somewhat tedious, but quite straightforward to substitute decompositions corresponding to (5.18) in place of the imaginary parts $I_{\mu \lambda}$ and $I_{\lambda_{\nu}}$ in Eq. (5.20), and to evaluate the averages $\left\langle l_{\mu}\right\rangle$ and $\left\langle l_{\mu} l_{\nu}\right\rangle$ which occur. Anticipating the form which the induction argument will take, it is evident that we may expect to have to evaluate integrals like the one in Eq. (5.13), but with the integrand multiplied by various types of factors from the spin analysis. Actually carrying out the detailed algebra shows that these additional factors are $t, t_{1}, t_{2}$, or $t_{1} t_{2} / t$. It is easy to find out the large $t$ behavior of the resulting integrals:

$$
\begin{gathered}
\int d t_{1} \int d t_{2} K\left(s, t, t_{1}, t_{2}\right) \frac{\left(\ln t_{1}\right)^{m-2}}{t_{1}} \frac{\left(\ln t_{2}\right)^{n-m-2}}{t_{2}} \times\left\{t, t_{1}, t_{1} t_{2} / t\right\} \\
\sim\left\{(\ln t)^{n-2},(\ln t)^{n-3},(\ln t)^{n-4}\right\}
\end{gathered}
$$


when $t$ is large. The result, then, is that the leading terms in (5.22) are those in which the spinology contributes an explicit factor $t$.

If we now accept this, we can discard everything in (5.22) except terms involving $p_{1} \cdot p_{2}$; in terms of the representation (5.18), such factors can only arise from products involving $B, C$, or $G$. Furthermore, these products only give rise to $A, D, E$, and $F$-type terms. Explicitly, we obtain the relations (we suppress the index " 13 " on $\rho$ )

$$
\begin{aligned}
& \rho_{A}=\frac{t}{2} \int d t_{1} \int d t_{2} K\left\{4\left|I_{B}\right|^{2}(1-2 \beta+c)\right. \\
& \left.+2\left(I_{B}{ }^{*} I_{C}+I_{C}{ }^{*} I_{B}\right)(1-\beta)+\left|I_{C}\right|^{2}\right\}, \\
& \rho_{D}=\frac{t}{2} \int d t_{1} \int d t_{2} K\left\{16\left|I_{B}\right|^{2} d\right\}, \\
& \rho_{E}=\frac{t}{2} \int d t_{1} \int d t_{2} K\left(-8\left(I_{B}{ }^{*} I_{G}+I_{G}{ }^{*} I_{B}\right)\right. \\
& \left.+4\left|I_{G}\right|^{2}+16\left|I_{B}\right|^{2}\right\} \\
& \rho_{F}=\frac{t}{2} \int d t_{1} \int d t_{2} K\left\{8\left|I_{B}\right|^{2}(\beta-1)\right. \\
& -2\left(I_{B}{ }^{*} I_{C}+I_{C}{ }^{*} I_{B}\right)+2\left(I_{B}{ }^{*} I_{G}+I_{G}{ }^{*} I_{B}\right)(1-\beta) \\
& \left.+\left(I_{C}{ }^{*} I_{G}+I_{G}{ }^{*} I_{C}\right)\right\} \text {. }
\end{aligned}
$$

The notation $\rho_{A} \cdots \rho_{G}$ and $I_{A} \cdots I_{G}$ comes from representations of the weight functions $\rho_{\mu \nu}$ and the imaginary part $I_{\mu \nu}$ similar to (5.18) for the amplitude itself. $\beta, c$, and $d$ are spin factors resulting from the averages $\left\langle l_{\mu}\right\rangle$ and $\left\langle l_{\mu} l_{\nu}\right\rangle$; they are given by

$$
\beta=\frac{1}{2}, \quad c=\frac{1}{2}-(1 / s), \quad d=1-(s / 4) .
$$

As we have mentioned earlier, the seven amplitudes $A \cdots G$ are, of course, not independent. We may employ the relations (5.19) (or rather their analogs for $I_{A} \cdots I_{G}$, which are also valid) to express the right-hand sides of Eqs. (5.24) in terms of $I_{A}, I_{D}, I_{E}$, and $I_{F}$. We thus have four two-particle unitarity conditions, essentially analogous to the totally spinless case, except for the multiplicative factor $t / 2$ and the fact that the four independent amplitudes mix among themselves. The mixing may be minimized by choosing four new independent amplitudes, linear combinations of the old, which in effect diagonalize the unitarity conditions. If we define

$$
\begin{aligned}
& \mathfrak{M}_{1}=(1 / t)\left[2 s^{2} A+(s / 2) D+2 E+4 s F\right], \\
& \mathfrak{T}_{2}=(1 / t)[8 s A+2 D+2 E+(2 s+8) F], \\
& \mathfrak{M}_{3}=(1 / t)[32 A+(8 / s) D+2 E+16 F], \\
& \mathfrak{M}_{4}=(1 / t)[(4-s) / 2] D,
\end{aligned}
$$

with analogous definitions for the imaginary parts and weight functions, the asymptotic unitarity conditions
(5.24) become simply

$$
\begin{aligned}
& \rho_{1}=\int d t_{1} \int d t_{2} K\left\{I_{1}{ }^{*} I_{1}\right\} \\
& \rho_{2}=\int d t_{1} \int d t_{2} K\left\{\frac{1}{2}\left(I_{1}^{*} I_{2}+I_{2}{ }^{*} I_{1}\right)\right\} \\
& \rho_{3}=\int d t_{1} \int d t_{2} K\left\{I_{2}{ }^{*} I_{2}\right\} \\
& \rho_{4}=\int d t_{1} \int d t_{2} K\left\{I_{4}^{*} I_{4}\right\} .
\end{aligned}
$$

The first and fourth of these are precisely the same as spinless unitarity conditions.

Evidently, $\mathfrak{M}_{1}$ through $\mathfrak{T}_{4}$ are related to the f's defined in Sec. 2 by

$$
\begin{aligned}
& \mathfrak{M}_{1}=-8 \pi W f_{11}{ }^{(+)}, \\
& \mathfrak{M}_{2}=-(16 \pi / \sqrt{2}) f_{10}{ }^{(+)}, \\
& \mathfrak{M}_{3}=-8 \pi W[(s-4) / s t] f_{00}{ }^{(+)}, \\
& \mathfrak{T}_{4}=-8 \pi W f_{11}{ }^{(-)} .
\end{aligned}
$$

\section{THE PERTURBATION SERIES: SCALAR-VECTOR COMPTON SCATTERING}

Let us now return to Eqs. (5.27) and proceed to "solve" them. ${ }^{6}$ The amplitude $\mathfrak{M}_{4}$ [which corresponds to $P(-1)^{J}=-1$ ] clearly does not couple to anything else, and the unitarity condition which it (asymptotically) satisfies is indistinguishable from the spinless case. The induction argument used there would, as a consequence, work equally well for $\mathfrak{T H}_{4}$, so we may conclude that

$$
\mathfrak{M T}_{4}^{(n)}=\left(C_{4}\right)^{n} \mathfrak{T}^{(n)},
$$

where $\mathfrak{T}^{(n)}$ is the $n$ th-order term (that is, the coefficient of $\gamma^{2 n}$ ) in the spinless problem, $\mathfrak{T T}_{4}{ }^{(n)}$ is the $n$ th-order term in $\mathfrak{M T}_{4}$, and $C_{4}$ is some function of $s$ independent of $n$. The unitarity condition expresses any order of perturbation theory in terms of lower orders, except of course the first. $C_{4}$ is thus in the way of being a constant to be determined by the initial conditions; that is, by the Born approximation.

The Born approximation diagrams for Compton scattering are shown in Fig. 9; corresponding to the

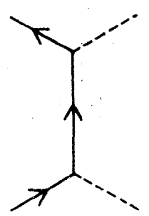

(a)

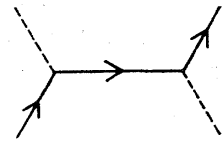

(b)

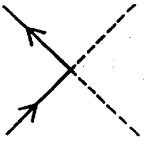

(c)
Frg. 9. Lowest order Compton scattering graphs.

${ }^{6}$ The actual solution of the asymptotic unitarity equations is completely analogous to the work of Sec. 2 . However, we include it in order to show how the method can be carried to a conclusion. 
three figures we have the lowest order amplitude (already calculated in Sec. 2)

$$
\begin{aligned}
\mathfrak{M}_{\mu \nu} & =-\left[\gamma^{2} /(s-1)\right]\left(2 p_{2}+k_{2}\right)_{\mu}\left(2 p_{1}+k_{1}\right)_{\nu} \\
& -\left[\gamma^{2} /(u-1)\right]\left(2 p_{2}-k_{1}\right)_{\mu}\left(2 p_{1}-k_{2}\right)_{\nu}-2 \gamma^{2} \delta_{\mu \nu} .
\end{aligned}
$$

The current conservation $\mathfrak{T}_{\mu \nu} k_{1 \nu}=0$ may be checked explicitly here. Note that the "seagull" picture, Fig. 9(c), is essential for current conservation and, consequently, also for renormalizability.

From (6.2), the Born approximation contributions to the amplitudes $A$ through $G$ may be extracted; we have

$$
\begin{aligned}
& A=-\gamma^{2} /(s-1), \\
& B=-\gamma^{2} /(u-1), \\
& C=0, \\
& D=2 \gamma^{2}, \\
& E=-\left[\gamma^{2} /(s-1)\right]-\left[\gamma^{2} /(u-1)\right], \\
& F=\gamma^{2} /(s-1), \\
& G=-\gamma^{2} /(u-1) .
\end{aligned}
$$

The calculation of the lowest order contributions to $\mathfrak{N}_{1}$ through $\mathfrak{N C}_{4}$ is now trivial, and we get

$$
\begin{aligned}
& \mathfrak{M}_{1}^{(1)}=\left(\gamma^{2} / t\right) \cdot(s-2), \\
& \mathfrak{M}_{2}^{(1)}=\left(\gamma^{2} / t\right) \cdot(2), \\
& \mathfrak{M}_{3}{ }^{(1)}=\left(\gamma^{2} / t\right) \cdot[(2 s+16) / s(s-1)], \\
& \mathfrak{M}_{4}{ }^{(1)}=\left(\gamma^{2} / t\right)\{[(s-4) / 2] .
\end{aligned}
$$

Going back to (6.1), we may use the fact that in the spinless case $\mathfrak{T}^{(1)}=\gamma^{2} / t$ for large $t$, together with (6.4), to derive

$$
C_{4}=\frac{\mathscr{T}_{4}{ }^{(1)}}{\mathscr{T}^{(1)}}=\frac{\left(\gamma^{2} / t\right)(s-4)}{\left(\gamma^{2} / t\right)}=(s-4) ;
$$

then we get

$$
\mathfrak{M T}_{4}{ }^{(n)}=\left[(s-4)^{n}\right] \mathscr{M}^{(n)} .
$$

Since $\mathfrak{T}^{(n)}$ itself was given by a Regge form, Eq. (5.10), with

$$
\alpha(s)=\frac{\gamma^{2}}{8 \pi^{2}} \frac{1}{[s(s-4)]^{1 / 2}} \ln \frac{(s-4)^{1 / 2}+s^{1 / 2}}{(s-4)^{1 / 2}-s^{1 / 2}},
$$

we now find for $\mathfrak{M}_{4}$ [as in (2.8)] a Regge behavior but with a trajectory

$$
\alpha^{-}(s)=\frac{\gamma^{2}}{8 \pi^{2}} \frac{s-4}{[s(s-4)]^{1 / 2}} \ln \frac{(s-4)^{1 / 2}+s^{1 / 2}}{(s-4)^{1 / 2}-s^{1 / 2}} .
$$

The $P(-1)^{J}=-1$ problem is naturally completely uncoupled from the $P(-1)^{J}=1$ problem, so we may now abandon it completely, and turn to $\mathfrak{N T}_{1}, \mathfrak{N T}_{2}$, and $\mathfrak{M}_{3}$ which describe the $P(-1)^{J}=1$ channels. $\mathfrak{N}_{1}$, we notice, satisfies the spinless unitarity condition; hence, we may write

$$
\mathfrak{M}_{1}^{(n)}=\left(C_{1}\right)^{n} \mathfrak{M C}^{(n)}
$$

just as we did for $\mathfrak{M}_{4}$. $C_{1}$ is now found by matching the "initial condition," Eq. (6.4), to be

$$
C_{1}=\frac{\mathfrak{M}_{1}^{(1)}}{\mathfrak{N}^{(1)}}=\frac{\left(\gamma^{2} / t\right)(s-2)}{\left(\gamma^{2} / t\right)}=(s-2)
$$

so we get that $\mathfrak{M}^{(1)}$ as in (2.9) also has the Regge form with a trajectory

$$
\alpha^{+}(s)=\frac{\gamma^{2}}{8 \pi^{2}} \frac{(s-2)}{[s(s-4)]^{1 / 2}} \ln \frac{(s-4)^{1 / 2}+s^{1 / 2}}{(s-4)^{1 / 2}-s^{1 / 2}}
$$

which, as remarked earlier, does not pass through the nucleon.

In the unitarity condition for $\mathfrak{M}_{2}$, we have a linear equation, since $\rho_{2} \sim I_{2} I_{1}$ rather than $I_{2}{ }^{2}$. The solution here is then

$$
\mathfrak{M C}_{2}^{(n)}=C_{2} \mathfrak{M}_{1}^{(n)}=C_{2}\left(C_{1}\right)^{n} \mathfrak{T}^{(n)} .
$$

Comparing with lowest order again, we get

$$
C_{2}=\frac{\mathfrak{T}_{2}^{(1)}}{C_{1} \mathfrak{T}^{(1)}}=\frac{\left(\gamma^{2} / t\right)(2)}{(s-2)\left(\gamma^{2} / t\right)}=\frac{2}{s-2} .
$$

$\mathfrak{N}_{2}$ then also behaves in the Regge manner, and of course with the same trajectory $\alpha^{+}$. Finally, $\mathfrak{M}_{3}$ can be seen from $(5.27 \mathrm{~d})$ to be expressible as

$$
\mathfrak{M}_{3}{ }^{(n)}=C_{2}{ }^{2} \mathfrak{M}_{1}{ }^{(n)}=C_{2}{ }^{2}\left(C_{1}\right)^{n} \mathfrak{M}^{(n)} \text {. }
$$

There is now no arbitrary constant, so we cannot guarantee that the lowest order term will fit onto this formula. It either will or will not, and according as it does or doesn't, the entire amplitude is or is not expressible as a Regge trajectory.

Comparing (6.13) and (6.11), we see that we get a factorizing law,

$$
\mathfrak{T}_{1}{ }^{(n)} \mathfrak{T}_{3}{ }^{(n)}=\left(\mathfrak{M}_{2}{ }^{(n)}\right)^{2}
$$

and in particular this must be true of lowest order if everything is to fit onto the Regge trajectory. In lowest order,

$$
\mathfrak{M}_{1}^{(1)} \mathfrak{M}_{3}{ }^{(1)}=\left[\frac{(s-2)(2 s+16)}{s(s-1)}\right]\left(\frac{\gamma^{2}}{t}\right)^{2}
$$

and this is manifestly unequal to $\left(\mathscr{T}_{2}{ }^{(1)}\right)^{2}$. The conjecture that the perturbation theory sums to a Regge pole thus fails, as we have already seen.

It is not hard to see what the actual sum is; the only thing that went wrong was that the lowest order expression for $\mathfrak{T}_{3}$ did not fit onto the general form required by unitarity. If we rewrite the lowest order $\mathfrak{M}_{3}$ as

$$
\left[\mathfrak{M}_{3}{ }^{(1)}-\frac{\left(\mathfrak{M}_{2}^{(1)}\right)^{2}}{\mathfrak{M}_{1}{ }^{(1)}}\right]+\frac{\left(\mathfrak{M}_{2}{ }^{(1)}\right)^{2}}{\mathfrak{M}_{1}{ }^{(1)}},
$$

then the second term does fit on, and the first is just left hanging. The entire result, then, is just a Regge 
pole with trajectory $\alpha^{+}(s)$, appearing in $\mathfrak{M}_{1}, \mathfrak{M}_{2}$, and $\mathfrak{M}_{3}$ as given by Eqs. (6.8), (6.11), and (6.13), plus $\mathfrak{M}_{3}{ }^{(1)}-\left(\mathfrak{M}_{2}{ }^{(1)}\right)^{2} / \mathfrak{M}_{1}{ }^{(1)}$ in the $\mathfrak{M}_{3}$ term. Explicitly, we get, as $t \rightarrow \infty, 7$

$$
\begin{aligned}
& \mathfrak{M C}_{1} \rightarrow\left(\gamma^{2} / 2 t\right)(s-2)\left\{\left[(-t)^{\alpha^{+}}+t^{\alpha^{+}}\right]\right. \\
& \left.-\left[(-t)^{-\alpha^{+}}-t^{-\alpha^{+}}\right]\right\}
\end{aligned}
$$

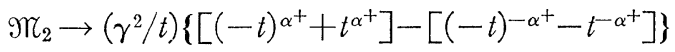

$$
\begin{aligned}
& \mathfrak{N}_{3} \rightarrow\left(\gamma^{2} / t\right)\left[\frac{2}{s-2}\right]\left\{\left[(-t)^{\alpha^{+}}+t^{\alpha^{+}}\right]-\left[(-t)^{-\alpha^{+}}-t^{-\alpha^{+}}\right]\right\} \\
& +\frac{\gamma^{2}}{t}\left[\frac{2 s+16}{s(s-1)}-\frac{4}{s-2}\right]
\end{aligned}
$$

where $\alpha^{+}$is given by (5.10). For $\mathfrak{M}_{4}$, of course, we have $\mathfrak{M}_{4} \rightarrow\left(\gamma^{2} / 2 t\right)(s-4)\left[\left[(-t)^{\alpha^{-}}+t^{-}\right]-\left[(-t)^{-\alpha^{-}}-t^{-\alpha^{-}}\right]\right]$

with $\alpha^{-}$defined by (6.7). The original amplitudes, $A \cdots G$, in terms of which we wrote the scattering amplitude $\mathfrak{T}_{\mu \nu}$, are easily obtained using the definitions given in Eqs. (5.26). Note that the nucleon pole at $s=1$ appears in the term hanging on the end of $\Re_{3}$ and not in the Regge-like pieces. This is just what one should expect on the basis of the idea that "elementary" particles

7 Note that our induction argument of course gives us the weight function, not the amplitude itself. The expressions wc write throughout this section for the computed amplitudes are valid only if the generalization of the " $\log ^{2}$ " cancellation of Sec. 3 holds. We are not able to show in general that it does. do not appear as Regge poles but as fixed Kronecker deltas in angular momentum.

\section{CONCLUSIONS}

In our approximation we have found, for photonscalar nucleon scattering in the usual field theory, Regge pole terms plus a term of fixed angular momentum.

In the channel with $P(-1)^{J}=-1$, there are two Regge poles of opposite signature and (in second order) equal and opposite values of $\alpha$.

In the channels with $P(-1)^{J}=1$, there are likewise two Regge poles of opposite signature. For positive signature, we find, for equal masses, the second-order value

$$
\alpha^{+}(s)=\frac{\gamma^{2}}{8 \pi^{2}} \frac{s-2}{[s(s-4)]^{1 / 2}} \ln \frac{(s-4)^{1 / 2}+s^{1 / 2}}{(s-4)^{1 / 2}-s^{1 / 2}} .
$$

Again the negative-signature trajectory has an equal and opposite value in second order.

Because of the failure of the Born approximation to factor, the nucleon does not lie on the trajectory (7.1). Instead, there is a $\delta_{J 0}$ term in the partial wave amplitudes with a pole in (energy) ${ }^{2}$ at the square of the nucleon mass.

If a special extra coupling is introduced into the theory, the Born approximation can be modified so that it does factor, and the scalar nucleon does then lie on the appropriate trajectory, but it is not clear that the resulting theory can be renormalized. 